\title{
Conservative Management of an Advanced Abdominal Pregnancy at 22 Weeks
}

\author{
Louis Marcellin, MD ${ }^{1,2}$ Sophie Ménard, MD ${ }^{1,2}$ Marie-Charlotte Lamau, MD \\ Alexandre Mignon, MD, $\mathrm{PhD}^{3,4}$ Marie Stephanie Aubelle, MD ${ }^{2,5}$ Gilles Grangé, MD ${ }^{1,2}$ \\ François Goffinet, MD, $\mathrm{PhD}^{1,2}$
}

${ }^{1}$ Maternité Port-Royal, Groupe hospitalier Cochin, Broca, Hôtel-Dieu, Assistance Publique-Hôpitaux de Paris, Paris, France

${ }^{2}$ DHU Risques et Grossesse, Université Paris Descartes, Paris, France

3 Université Paris Descartes, Assistance Publique-Hôpitaux de Paris,

Paris, France

${ }^{4}$ Department of Anaesthesia and Critical Care Paris, Cochin University Hospital, Paris, France

${ }^{5}$ Service de Médecine et Réanimation néonatales de Port-Royal, Groupe hospitalier Cochin, Broca, Hôtel-Dieu, Assistance PubliqueHôpitaux de Paris, Paris, France

Address for correspondence Louis Marcellin, MD, Maternité Port Royal, Université Paris Descartes, 53 avenue de l'Observatoire, 75014 Paris, France (e-mail: louis.marcellin@cch.aphp.fr).

Am J Perinatol Rep 2014;4:55-60.

\begin{abstract}
Keywords

- advanced abdominal pregnancy

- preterm delivery

- placental embolization

Objective We report an uneventful conservative approach of an advanced abdominal pregnancy discovered at 22 weeks of gestation.

Study Design This study is a case report.

Results Attempting to extend gestation of an advanced abdominal pregnancy is not a common strategy and is widely questioned. According to the couple's request, the management consisted in continuous hospitalization, regular ultrasound scan, and antenatal corticosteroids. While the woman remained asymptomatic, surgery was planned at 32 weeks, leading to the birth of a preterm child without any long-term complications. Placenta was left in situ with a prophylactic embolization, and its resorption was monitored.

Conclusion Depending on multidisciplinary cares and agreement of the parents, when late discovered, prolonging advanced abdominal pregnancy appears to be a reasonable option.
\end{abstract}

Abdominal pregnancy refers to a pregnancy that has been implanted in the peritoneal cavity, outside the uterine cavity and the fallopian tubes ${ }^{1}$ and represents $1 \%$ of ectopic pregnancies. ${ }^{2}$ In contrast to tubal ectopic pregnancies, abdominal pregnancies are usually asymptomatic at the early stage, may be discovered later on, and can reach an advanced gestational age with an incidence ranging from 1 of 1,320 births in nonindustrialized countries to 1 of 10,200 births in industrialized ones. ${ }^{3}$ Commonly, termination of pregnancy is decided in such condition, to avoid maternal risks of a pregnancy's continuation. ${ }^{4}$ However, the therapeutic strategy is not straightforward. We present here a case of an abdominal pregnancy discovered at 22 weeks of pregnancy, which could be extended uneventfully until 32 weeks. Management of abdominal pregnancy is discussed.

\section{Case Report}

A 24-year-old Caucasian woman, gravida 2 para 0 , who had a history of uncomplicated surgical abortion during the current year, was referred to our center at 22 weeks of pregnancy for an abdominal located pregnancy. Despite a first 18 weeks received

November 14, 2013 accepted after revision January 20, 2014 published online April 2, 2014
DOI http://dx.doi.org/ 10.1055/s-0034-1371749. ISSN 2157-6998.
Copyright (c) 2014 by Thieme Medical Publishers, Inc., 333 Seventh Avenue, New York, NY 10001, USA. Tel: +1(212) 584-4662.
License terms

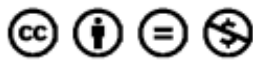



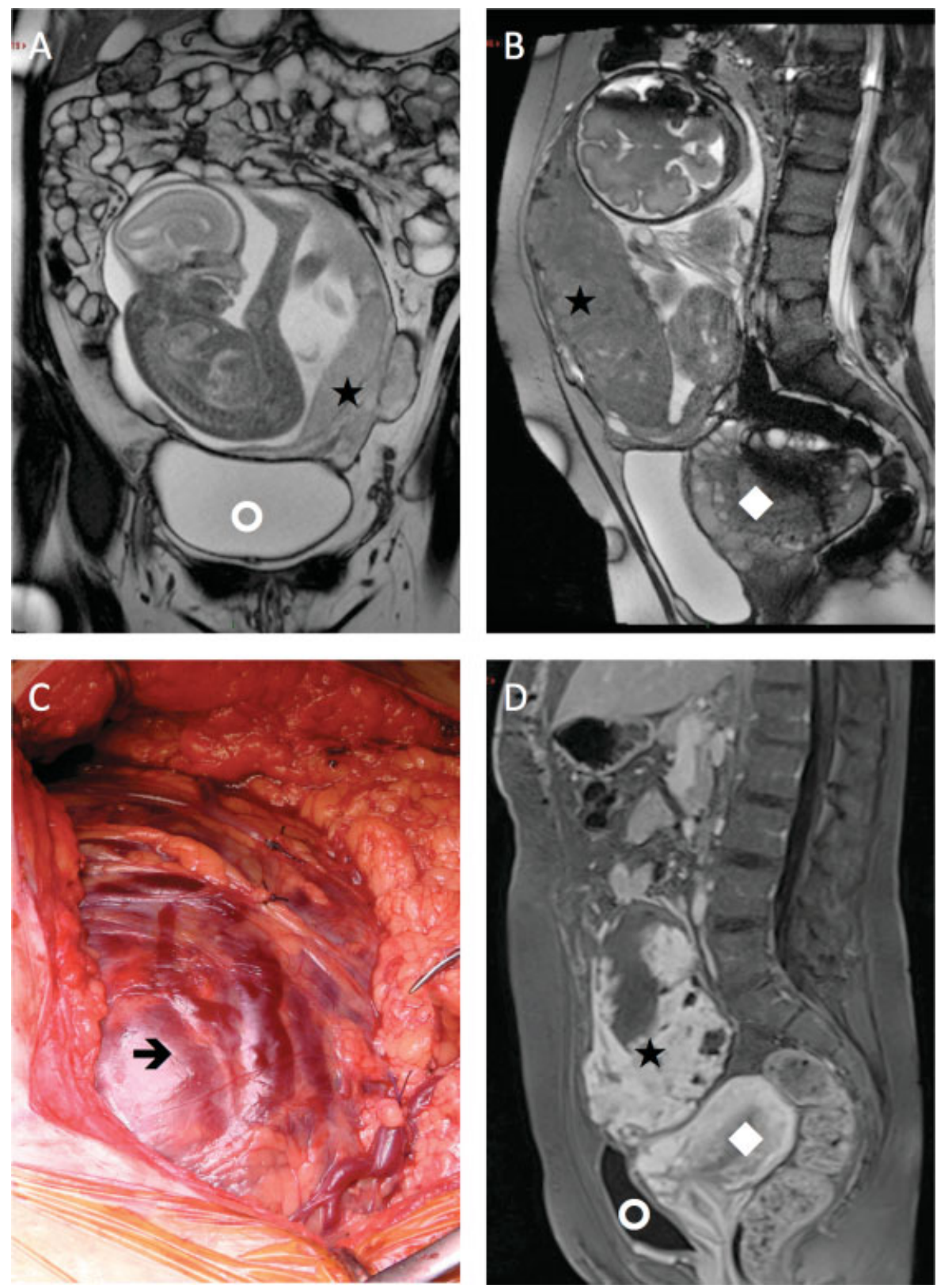

Fig. 1 Magnetic resonance imaging (MRI) and per operative view of an advanced abdominal pregnancy. (A) 23 weeks of gestation T2-weighted MRI sequence coronal view: the gestational sac is within the bowel loops with no myometrial wall. The left side of the placenta is seen $\left({ }^{*}\right)$, with integrity of the posterior wall of the bladder (white circle). (B) A 29 weeks of gestation T2-weighted MRI sequence sagittal view. The pregnancy is located above the uterus (white diamond). The placenta is anterior with apparent no invasion of the parietal wall (*). (C) Preoperative view of the subumbilical laparotomy. The gestational sac is covered with vascular plexuses. Amniotomy was done in an area free of them, away from the placenta insertion (black arrow). (D) Two weeks postoperative T1-weighted MRI sequence sagittal view. The placenta left in situ is being involute above the uterus (white circle). The bladder is not affected by placenta (white diamond).

ultrasonography considered as normal, the routine second trimester ultrasonography performed at 22 weeks identified an intra-abdominal eutrophic fetus free of any morphological abnormality, with a normal amniotic fluid quantification. Magnetic resonance imaging (MRI) with gadolinium injection, confirmed the abdominal location of the gestational sac, with a left anterior placenta inserted along the abdominopelvic peritoneum without sign of adhesion with the sigmoid and the left colon. The uterus and the cervix were normal, with usual measurements expected in a nonpregnant wom- an. Placental vascularization was mainly originating from a large branch of the left uterine artery, and moderate venous vascularization (-Fig. 1A, B). Life-threatening risks of an expectant management, with the aim of reaching a viable gestational age to give birth to a healthy child, were thoroughly explained to the couple. Initially, a termination of pregnancy with scheduled surgical extraction was suggested. Despite the risks, the couple chose to extend the pregnancy in the hope to get a healthy child. The patient was hospitalized up to the 32nd week of gestation, with ultrasonography 
performed twice a month. Fetal maturation was enhanced with two corticosteroids therapies administered at 24 and 30 weeks of gestation. The pregnancy follow-up was uncomplicated: the woman did not complain of any symptoms throughout hospital stay, and fetal growth was regular with normal amniotic fluid quantification and Doppler investigations. A multidisciplinary consultation, including obstetricians, general surgeons, anesthesiologists, pediatricians, and radiologists, led us to schedule a surgical preterm extraction at 32 weeks of gestation. Preoperatively, anesthetic management had been anticipated during a specific consultation with pretransfusional check and appropriate blood products storing. Before intervention, an invasive and continuous blood pressure monitoring had been set. Surgery was performed under general anesthesia. Preoperative hemoglobin was $12 \mathrm{~g} /$ $\mathrm{dL}$ with normal hemostatic function.

A final accurate ultrasound location of the placenta was done in the operative room just before incision. A median vertical laparotomy was performed with a xipho-ombilical incision. After opening the parietal fascia, an unusual thickness of the peritoneum ( $\mathbf{- F i g . 1 C}$ ) was observed and carefully dissected to reach the abdominal cavity. The gestational sac was identified, easily cleavable from the peritoneum, partially covered with vessels and omental adhesions. After progressive dissection of the omentum, the amniotic cavity was opened through an avascular area of the gestational sac that enabled a careful cephalic extraction of a 2,300-g girl, without any malformation, immediately managed by neonatologists. She initially had a worst adaptation to extrauterine life requiring endotracheal ventilation for 6 hours, supposed to be link to the prolonged general anesthesia of her mother before delivery.

We did not attempt any placental delivery or traction on the umbilical cord. The umbilical cord was rather completely purged and ligated at its proximal insertion. Cautious exploration of the cavity did not reveal any active bleeding and placental structure was left in situ, with a redon drainage placed in the amniotic cavity and another one in the abdominal cavity. Each layer of the abdominal wall was sewn up. Despite appropriate anticipation of massive blood loss (cell salvage and blood products storage), no hemorrhagic complication was observed during both surgical procedure and immediate postoperative follow-up. A preoperative antibiotic therapy with ceftazidime $1 \mathrm{~g}$ was administered. A postoperative arteriography, realized immediately after surgery, did neither show any active bleeding nor aberrant mesenteric vessels. Most of the placental vascularization was detected in projection of the left periumbilical region. Selective catheterization of the left uterine artery revealed a classical uterine vasculature in the lower part, and a large artery leading to the placenta in the upper part, without any arteriovenous fistula. A selective embolization of this large arterial ramification was performed with microspheres that allowed a selective devascularization of the placenta (first with 2 syringes of 300$500 \mu \mathrm{m}$ microspheres, then 4 syringes $500-700 \mu \mathrm{m}$, and finally with 11 syringes $900-1,200 \mu \mathrm{m}$ ). Selective catheterization of the right uterine did not show any abnormal vascularization.
The patient was supervised at the hospital during 15 days without any complication. The redon drainage were removed the 4th day after surgery. Before leaving the hospital, the woman was asymptomatic, with a normal hemoglobin level $(11.1 \mathrm{~g} / \mathrm{dL})$ and a decreasing inflammatory syndrome (Creactive protein, $62 \mathrm{mg} / \mathrm{L}$ and leukocytosis, 9,400/( $\mathrm{mm}^{3}$ ). The neonate was performed in the neonatal care unit during 4 weeks before going home. She had a regular growth and normal development, excepting an axial hypotonia, and her weight was 2,800 g prior hospital discharge.

An early postoperative ultrasound control of the placenta showed a homogeneous mass, $126 \times 97 \times 124 \mathrm{~mm}$ in size, movable in the abdominal cavity respecting the bladder integrity. Doppler examination revealed that the placenta was vascularized with a low resistance (resistance index [RI], 0.22 ) and a high $C_{\max }(90 \mathrm{~cm} / \mathrm{s})$ by a main placental artery originated from the left uterine artery. Size and structure of the placenta left in situ were also observed at MRI ( - Fig. 1D).

Regular clinical and paraclinical follow-up were performed twice a month at the beginning. The patient remained fully asymptomatic with a persistent biological inflammatory syndrome during the first 3 months following surgery and delivery. Placental mass volume, estimated by measuring the three higher diameters in the three plans, decreased gradually: approximately $20 \%$ after 3 months and by half after 7 months of the postoperative follow-up period (-Fig. 2A). Left uterine artery and placental vascularization from the left uterine artery branch had both increasing resistance index with decreased $\mathrm{C}_{\max }$ after 3 months (-Fig. 2 B, C). After 15 months, the placental structures were almost unrecognizable, described as a sluggish pelvic mass $2 \times 2 \times 3 \mathrm{~cm}$ in size, located above the uterus ( - Fig. 2D). The woman and her daughter have been seen 1 year later in good health.

\section{Discussion}

Advanced abdominal pregnancy may remain paucisymptomatic for a long time and women can reach an advanced gestational age before accurate diagnosis. When discovered during the third trimester, a surgical delivery can be scheduled with restrained fetal impact. Conversely, a discovery at 22 weeks is a specific condition that raised the opportunity of an expectative approach during several weeks despite maternal life-threatening risk exposure. This strategy could be considered to reach a gestational age compatible with viability.

Prolongation of advanced abdominal pregnancy remains controversial. Surgical termination of pregnancy should be proposed as soon as possible, when operative security conditions are guaranteed. ${ }^{4}$ Several teams have since described expectant approaches when faced with the couples rejecting a termination of pregnancy. Beddock et al in 2004 report an abdominal pregnancy discovered during second trimester, which ended successfully at 37 weeks of gestation without any fetal or maternal complication. ${ }^{5}$ Conversely, Bertrand et al recently failed to prolong an abdominal pregnancy discovered at 17 weeks, which complicated with a major hemoperitoneum few days later. A 

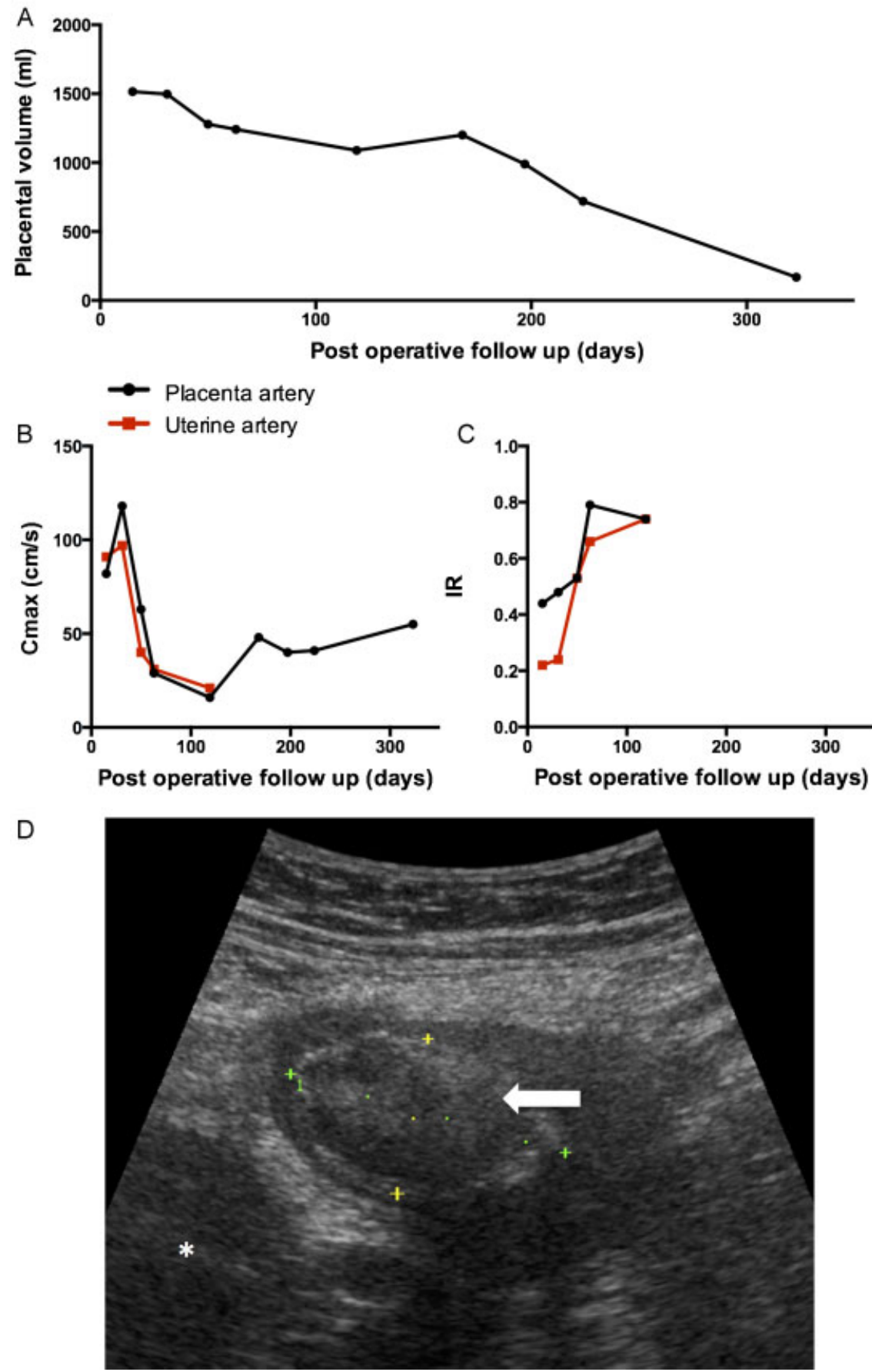

Fig. 2 (A) Postoperative ultrasound follow-up of the placenta (days). Theoretical volume evolution of the placenta: the decrease of placenta volume is regular during the 1 st year postoperative period. (B) $C_{\max }$ and (C) resistance index in Doppler analyze of placental artery (black) and left uterine artery (red): vascular parameter changed within the first trimester in postoperative period with a decrease of the $C_{\max }$ and an increase of the resistance index. (D) Sonographic view of the placenta after 15 months, showing a pelvic mass (white arrow) located above the uterus ( ${ }^{*}$ ). IR, resistance index.

termination of the pregnancy was decided: preoperative embolization of the uterine arteries was performed before the emergency laparotomy. This clinical management saved the patient. Placenta was also left in situ. ${ }^{6}$ According to the literature, expectant attitude could be considered in exceptional condition, only if, specified criteria are met (-Table 1). ${ }^{7}$ The asymptomatic state of our patient prompted us to extend the pregnancy, in accordance with the couple's wish. However, we did not extend it longer than 32 weeks, because we considered that ending the pregnancy would probably limit placental extensions.

General anesthesia and aggressive monitoring are usually recommended, considering the important preoperative risk of a brutal and abundant bleeding, a possible prolonged operative time, and the risk of major stress for the patient. All the techniques used for major surgery with high hemorrhagic risk are used, including maintenance of normothermia, monitoring the depth of anesthesia, and optimization of 
Table 1 Items for an expectant strategy of an advanced abdominal pregnancy (according to Martin and $\mathrm{McCaul}^{7}$ )

\begin{tabular}{|l|}
\hline Diagnosis after 20 WG \\
\hline Absence of fetal malformation \\
\hline Lack of maternal or fetal decompensation \\
\hline Continuous monitoring of fetal well-being \\
\hline $\begin{array}{l}\text { Low-placenta insertion in the abdomen away from the } \\
\text { liver and the spleen }\end{array}$ \\
\hline Enough amniotic fluid quantity \\
\hline Continuous follow-up in a level 3 unit \\
\hline Consent couple after risks information \\
\hline Multidisciplinary consultation \\
\hline
\end{tabular}

Abbreviation: WG, weeks of gestation.

blood volume and hemostasis to avoid the lethal triad of hypothermia, acidosis, and coagulopathy. ${ }^{8}$

The conservative surgical success is inherent to the preservation of placental integrity during the fetal delivery. In our case, median laparotomy was realized with a preoperative mark of the placenta borders during a preoperative ultrasound, enabling an amniotomy in a remote area of the placental insertion. Most authors recommend a complete deliverance to reduce the postoperative morbidity. ${ }^{4}$ However, this strategy remains controversial because of the potential cataclysmic hemorrhage, with a perioperative morbidity related to neighboring organs resections in $76 \%$ of case with $27 \%$ of hysterectomy. ${ }^{4,9}$

To our knowledge, preoperative embolization of dead or unviable fetuses and postoperative emergency hemostatic procedures have only been reported to date. ${ }^{10-12}$ Oneko et al identified nine advanced abdominal pregnancies between 20 and 42 weeks of gestation in a low-resource center between 1999 and 2007. The placentas were left completely in situ in five of the nine cases. None of them had embolization. All nine mothers survived, and seven fetuses died before delivery.

The required time to complete involution of the placenta is variable, ranging from weeks to several months, even years, ${ }^{13}$ during the year, without any complications. As the placenta is left in situ, we proposed for surveillance: physical examinations, detection for sepsis, and ultrasound evaluations of the placenta resorption with a Doppler examination twice a month at the beginning. No data are available on the benefit of prophylactic antibiotics during the postoperative period. Some authors recommend monitoring the beta human chorionic gonadotropin ( $\beta$-hCG) blood level decrease. Intriguingly, Bajo et al described a direct relationship between the reverse decline in $\beta$-hCG and the increased resistance index in the utero-ovarian and subplacenta vessels. ${ }^{14}$ However, most studies have limited oversight of this involution. Additional treatment with Methotrexate has been reported, but it seems to be associated with an increased risk of infections without improving the rapidity of the placental resorption, which is coherent with the effectiveness of this treatment only on cells in division. ${ }^{15}$

In conclusion, when discovered late, advanced abdominal pregnancy is a rare condition whose management complexity is related to the balance between the maternal life-threatening risk exposure and the pregnancy continuation to reach fetal viability. We think that a conservative attitude is not a satisfactorily option, but it could be considered according to the couple's willingness and depends on the placental location, vascularity, and relationship to the adjacent organs. Prophylactic immediate postoperative embolization of the placenta left in situ seems to be a suitable option to prevent a secondary hemorrhage.

\section{Acknowledgments}

We are indebted to the whole staff of the Port Royal Maternity for assistance in the management of the patient presented in this report. We also thank Ludivine Doridot for English editing of the manuscript.

\section{References}

1 Gayer G. Images in clinical medicine. Abdominal ectopic pregnancy. N Engl J Med 2012;367(24):2334

2 Farquhar CM. Ectopic pregnancy. Lancet 2005;366(9485): 583-591

3 Oneko O, Petru E, Masenga G, Ulrich D, Obure J, Zeck W. Management of the placenta in advanced abdominal pregnancies at an East african tertiary referral center. J Womens Health (Larchmt) 2010;19(7):1369-1375

4 Hallatt JG, Grove JA. Abdominal pregnancy: a study of twenty-one consecutive cases. Am J Obstet Gynecol 1985;152(4):444-449

5 Beddock R, Naepels P, Gondry C, et al. [Diagnosis and current concepts of management of advanced abdominal pregnancy]. Gynecol Obstet Fertil 2004;32(1):55-61

6 Bertrand G, Le Ray C, Simard-Emond L, Dubois J, Leduc L. Imaging in the management of abdominal pregnancy: a case report and review of the literature. J Obstet Gynaecol Can 2009; 31(1):57-62

7 Martin JN Jr, McCaul JF IV. Emergent management of abdominal pregnancy. Clin Obstet Gynecol 1990;33(3):438-447

8 Rackelboom T, Tsatsaris V, Silvera S, Vignaux O, Goffinet F, Mignon A. Anomalies d'insertion placentaire: prise en charge anesthésique. Le Praticien en Anesthésie Réanimation 2011; 15(6):359-366

9 Hreshchyshyn MM, Bogen B, Loughran $\mathrm{CH}$. What is the actual present-day management of the placenta in late abdominal pregnancy? Analysis of 101 cases. Am J Obstet Gynecol 1961; 81:302-317

10 Cardosi RJ, Nackley AC, Londono J, Hoffman MS. Embolization for advanced abdominal pregnancy with a retained placenta. A case report. J Reprod Med 2002;47(10):861-863

11 Kerr A, Trambert J, Mikhail M, Hodges L, Runowicz C. Preoperative transcatheter embolization of abdominal pregnancy: report of three cases. J Vasc Interv Radiol 1993;4(6):733-735

12 Rahaman J, Berkowitz R, Mitty H, Gaddipati S, Brown B, Nezhat F. Minimally invasive management of an advanced abdominal pregnancy. Obstet Gynecol 2004;103(5 Pt 2):1064-1068

13 Valenzano M, Nicoletti L, Odicino F, Cocuccio S, Lorenzi P, Ragni N. Five-year follow-up of placental involution after abdominal pregnancy. J Clin Ultrasound 2003;31(1):39-43

14 Bajo JM, Garcia-Frutos A, Huertas MA. Sonographic follow-up of a placenta left in situ after delivery of the fetus in an abdominal pregnancy. Ultrasound Obstet Gynecol 1996;7(4):285-288

15 Skubisz MM, Tong S. The evolution of methotrexate as a treatment for ectopic pregnancy and gestational trophoblastic neoplasia: a review. ISRN Obstet Gynecol 2012;2012:637094 